\title{
Elaboración de un plan estratégico informático para el apoyo de los procesos administrativos en instituciones educativas
}

\section{Preparation of a strategic information plan to support administrative processes in educational institutions}

\author{
ROJAS, Luis P. ${ }^{1}$ \\ MARTELO, Raúl J. ${ }^{2}$ \\ FRANCO, David A. ${ }^{3}$
}

\section{Resumen}

El objetivo fue elaborar un plan estratégico informático (PEI) para el apoyo de procesos administrativos en instituciones educativas. Esta investigación fue de tipo cualitativa dado el tipo de manejo de los datos. La población estuvo conformada por 5 empleados del área administrativa de la institución educativa Cuarta Poza de Manga (Colombia). Como resultado se obtuvieron diferentes proyectos como organización documental, y capacitaciones técnicas, los cuales pueden reducir dificultades en procesos administrativos de la institución analizada.

Palabras clave: PEI, administración, institución educativa, TIC

\begin{abstract}
The objective was to develop a strategic information plan (PEI) to support administrative processes in educational institutions. This research was qualitative because the source of data. The population was made up of 5 employees from the administrative area of the Cuarta Poza de Manga (Colombia) educational institution. As a result, different projects were obtained such as document organization, and technical training, which can reduce difficulties in administrative processes of the analyzed institution.
\end{abstract}

Key words: SIP, administration, educational institution, ICT

\section{Introducción}

Una institución educativa es un sitio donde personas de diferentes edades pueden adquirir una formación académica (Shahid \& Luo, 2019) útil para el desempeño laboral. La operación de este tipo de entidades en el mercado contribuye la obtención de ventajas respecto al desarrollo sostenible de una nación (Labas, Darabos, \& Nagy, 2016), por lo cual se convierten en un mecanismo efectivo para el logro de cambios importantes en la sociedad. Existen diferentes dependencias en las cuales se basa una institución educativa, como la de recursos humanos, la académica, y la administrativa, las cuales cumplen con funciones particulares que contribuyen al alcance de los objetivos institucionales. Respecto a la última dependencia, se conforma por trabajadores

\footnotetext{
${ }^{1}$ Ingeniero de Sistemas. Facultad de Ingeniería. Universidad de Cartagena. e-mail:pedrolucho09@hotmail.com

2 PhD (e) - Magister en Informática. Líder del grupo de investigación INGESINFO. Profesor de planta del Programa Ingeniería de Sistemas de la Universidad de Cartagena. e-mail: rmartelog1@unicartagena.edu.co

${ }^{3}$ Magister en Ciencias Computacionales. Líder del grupo de investigación GIMATICA. Profesor de planta del Programa Ingeniería de Sistemas de la Universidad de Cartagena. e-mail: dfrancob@unicartagena.edu.co
} 
encargados de desempeñar actividades relacionadas con aspectos como la finanza, adquisición de bienes y servicios, mantenimiento, y almacenamiento de información administrativa de la institución (Almasri, Talla, AbuNaser, \& Shobaki, 2018).

Considerando la relevancia de este tipo de cargos en instituciones educativas, los empleados administrativos deben tener un buen rendimiento laboral con el fin de realizar los procesos de forma adecuada. No obstante, existe instituciones educativas donde la productividad de los trabajadores del área administrativa resulta afectada debido a las dificultades que se experimentan en la dependencia como el manejo poco efectivo de la información de manera y la utilización del papel como medio principal para el almacenamiento de la misma, el cual tiende al deterioro y puede extraviarse (Kumar \& Shardhi, 2019). Otra dificultad es la informalidad, debido a que algunas actividades de la dependencia administrativa, en diversas ocasiones, se realizan de manera verbal sin documentar debidamente la información generada en el proceso, lo cual puede reducir la efectividad al momento de efectuar procedimientos que requieran dicha información.

Una opción para reducir las dificultades es la planificación estratégica informática, debido a que esto permite analizar los procesos de la dependencia institucional analizada con el fin de establecer diferentes proyectos que permitan mejorar la gestión de la información mediante el uso de las tecnologías de la información y las comunicaciones (TIC) (Alamri, Almutiri, Ballahar, \& Zafar, 2016). La relevancia de la planificación estratégica informática se refleja en diferentes estudios, como el de Martelo y colaboradores (Martelo, Ponce, \& Acuña, 2015) donde se tomó a la Universidad de Cartagena como caso de estudio para la creación de un plan estratégico informático (PEI) y el de Sarif y colaboradores (Sarif, Rahman, Yunus, \& Rahamn, 2018) en el cual se crea un PEI para solventar necesidades informáticas en el ministerio de salud de Malasia. Otra investigación donde se evidencia es en el trabajo de Kitsios y colaboradores (Kitsios, Kamarituo, Madas, Fouskas, \& Manthou, 2020) donde se identifican factores que deben tener en cuenta los expertos al implementar una metodología para la creación de un PEI.

Teniendo en cuenta lo anterior, el propósito de esta investigación es elaborar un PEl para el apoyo de los procesos administrativos en instituciones educativas. Con la creación de un PEI para el área administrativa las instituciones educativas pueden incrementar el nivel competitivo y cumplir con los requerimientos del mercado.

\section{Metodología}

Esta investigación fue de tipo cualitativa debido a que se manejan datos de características cualitativas, es decir que no pueden ser medidos y que se representan mediante letras (Hernández, Fernández, \& Baptista, 2014). Considerando lo anterior, se adoptó un diseño de investigación fenomenológico debido a que se tienen en cuenta los puntos de vista que poseen diferentes personas sobre una situación específica en la cual están involucrados laboralmente (Cook, 2017).

\subsection{Población y muestra}

La población estuvo conformada por 5 empleados del área administrativa de la institución educativa Cuarta Poza de Manga. En cuanto a la muestra se aplicó un censo poblacional debido a que la población era finita y se contó con la facilidad para acceder a la totalidad de la misma.

\subsection{Técnicas de recolección de información}

Como técnica se implementó la entrevista con el fin de obtener información directa de los trabajadores que operan en el área administrativa de la institución. Además, se aplicó observación directa con el fin de recolectar información sobre los procesos del área analizada y la infraestructura tecnológica y de red. 


\subsection{Análisis de los datos}

Se crearon diferentes tablas con el fin de mostrar la información y facilitar la lectura y comprensión por parte del lector.

\subsection{Metodología para la elaboración del PEI}

Se utilizó como referente la metodología planteada en (Andrew, 1996) para la elaboración del PEI, donde se llevaron a cabo las siguientes etapas:

\subsubsection{Preparación y compromiso del equipo}

En esta etapa el experto selecciona un equipo de apoyo conformado, principalmente, por empleados de la institución analizada, a los cuales se les asignan responsabilidades en cuanto a la recolección de información en diferentes dependencias de la institución. Existen diversas situaciones en las cuales no es necesaria la creación de un equipo debido a que la empresa es pequeña o cuenta con pocos trabajadores, por lo cual el experto puede encargarse de recolectar la información.

\subsubsection{Descripción de la situación actual}

Con la información obtenida el experto se encarga de realizar una descripción de los procesos analizados a partir de la cual desarrolla diversas actividades como el diagnóstico estratégico, donde se analizan los procesos con el fin de dificultades en cuanto a las condiciones en las cuales son ejecutados; diagnóstico técnico, en el cual se verifica el funcionamiento de la infraestructura de red y los equipos; análisis de diagnóstico donde se evalúan las dificultades encontradas con el fin de identificar la prioridad de con la cual debe ser atendidas y proponer estrategias que permitan combatirlas.

\subsubsection{Elaboración del PEI}

En esta etapa se proponen proyectos conformados por estrategias y acciones con base en los inconvenientes a las cuales se desea dar solución. Luego, los directivos del ente analizado son convocados a una reunión en la cual se evalúan los proyectos establecidos con el fin de determinar viabilidad y aprobación.

\subsubsection{Programación de actividades}

Se asignan fechas en las cuales se llevarán a cabo los proyectos, el tiempo de duración y los recursos requeridos para su implementación.

\section{Resultados}

En esta sección se muestran los resultados obtenidos en las etapas que conforman el proceso de elaboración de un PEl.

\subsection{Preparación y compromiso del equipo}

No se consideró necesaria la creación de un grupo de apoyo, debido a que el área administrativa de la institución educativa Cuarta Poza de Manga cuenta con pocos trabajadores. Por lo anterior, los investigadores se encargaron de realizar la recolección de información personalmente.

\subsection{Descripción de la situación actual}

En esta etapa se realizó una descripción de la forma en la cual operan los trabajadores del área administrativa de la institución mediante diferentes actividades, a continuación se muestran las más relevantes.

\subsubsection{Identificación de los procesos}


Con la información obtenida a partir del paso anterior se realizó un análisis a través del cual se identificó la forma en la cual se encuentran organizados los procesos del área administrativa de la institución, como se observa en la Tabla 1.

Tabla 1

Identificación de los procesos

\begin{tabular}{|c|c|c|}
\hline Proceso & Sección & Subproceso \\
\hline \multirow[t]{4}{*}{ Gestión financiera } & \multirow[t]{4}{*}{$\begin{array}{l}\text { Sección de contabilidad y } \\
\text { presupuesto }\end{array}$} & $\begin{array}{l}\text { Identificación de necesidades institucionales en cuanto } \\
\text { a recursos y servicios }\end{array}$ \\
\hline & & $\begin{array}{l}\text { Elaboración del proyecto de presupuesto anual } \\
\text { institucional }\end{array}$ \\
\hline & & Ejecución y control presupuestal \\
\hline & & $\begin{array}{l}\text { Rendición de Cuentas y publicidad a la comunidad } \\
\text { educativa }\end{array}$ \\
\hline \multirow[t]{3}{*}{$\begin{array}{l}\text { Adquisición de bienes y } \\
\text { servicios }\end{array}$} & $\begin{array}{l}\text { Sección de compras y } \\
\text { adquisición }\end{array}$ & Compra de bienes y servicios \\
\hline & \multirow[t]{2}{*}{ Sección de almacén } & Recibo y control de bienes en la Institución \\
\hline & & Entrega de insumos a docentes y directivos \\
\hline \multirow[t]{2}{*}{ Soporte tecnológico } & \multirow[t]{2}{*}{ No existe } & $\begin{array}{l}\text { Gestión de solicitud de recursos tecnológicos para } \\
\text { actividades pedagógicas }\end{array}$ \\
\hline & & $\begin{array}{l}\text { Plan de mantenimiento a infraestructura tecnológica del } \\
\text { área administrativa }\end{array}$ \\
\hline
\end{tabular}

Fuente: Autores

Con base en lo anterior, entre los subprocesos más relevantes se destacan Identificación de necesidades institucionales en cuanto a recursos y servicios, Elaboración del proyecto de presupuesto anual institucional, Compra de bienes y servicios, Entrega de insumos a docentes y directivos, Gestión de solicitud de recursos tecnológicos para actividades pedagógicas, y Plan de mantenimiento a infraestructura tecnológica del área administrativa, los cuales se detallan a continuación.

Identificación de necesidades institucionales en cuanto a recursos y servicios: En este procedimiento se identifican las necesidades de la institución en cuanto a bienes y servicios para determinar requisitos financieros y elaborar el proyecto de presupuesto anual. Además, se definen los insumos que se van a obtener mediante el fondo de servicios educativos que administra la institución. Para llevar a cabo este subproceso el coordinador debe elaborar un documento en papel donde se notifique a los docentes sobre la reunión de autoevaluación institucional, la cual se efectúa cuando concluye el año escolar. La información obtenida con esta actividad se registra mediante papel y se almacena en archivadores localizados en rectoría.

Elaboración del proyecto de presupuesto anual institucional: El rector realizar una solicitud verbal al contador con el fin de que realice el proyecto de presupuesto anual, teniendo en cuenta las necesidades determinadas en la reunión de autoevaluación institucional. Posteriormente, el contador utiliza la aplicación Excel para realizar el proyecto de presupuesto anual, el cual es impreso y enviado a la oficina de rectoría. De esta forma, se lleva a cabo una reunión en el primer trimestre del año escolar con los empleados que conforman el Consejo Directivo de la institución, con el fin de evaluar el proyecto de presupuesto y aprobarlo. Cuando el documento es aprobado, se almacena en la carpeta de presupuestos y se guarda en un archivador.

Compra de bienes y servicios: El fin de este subproceso es efectuar la compra de bienes y servicios considerando los lineamientos de establecidos en el fondo de servicio educativo y la normativa legal que lo rige. Para dar inicio al subproceso, el coordinador o un profesor realiza una petición sobre la adquisición de un bien o servicio en particular. Luego, el rector verifica la disponibilidad de recursos financieros y toma una decisión respecto a la 
compra. Si el valor de la adquisición es mayor a los 20 SMMLV el rector debe obtener la aprobación del Consejo Directivo y tener en cuenta lo planteado en el estatuto contractual de la administración pública. Por último, cuando el rector recibe el bien comprado se entrega a los empleados a cargo de la bodega con el fin de guardarlo. La información generada con la realización de esta actividad se registra en papel y se almacena en archivadores localizados en la oficina de rectoría.

Recibo y control de bienes en la Institución: La finalidad de este procedimiento es recibir y manejar los materiales e insumos de la institución. El encargado de lo anterior es el empleado responsable de la bodega, no obstante, en diversas ocasiones estas funciones son ejecutadas por empleados ajenos al cargo, como el coordinador o el bibliotecario. La constancia recibida se registra en papel, pero en algunas ocasiones no es almacenado correctamente en los archivadores.

Entrega de insumos a docentes y directivos: El fin de este subproceso es la aprovisionar a la comunidad educativa con insumos necesarios para desempeñar de forma adecuada sus responsabilidades. Este procedimiento comienza con una petición al empleado encargado de la bodega sobre el insumo requerido, luego el trabajador entrega lo solicitado y registra la información en un cuaderno donde se estipulan datos relevantes como la fecha, el solicitante, y el insumo proporcionado.

Gestión de solicitud de recursos tecnológicos para actividades pedagógicas: El propósito de este procedimiento es administrar la instalación de equipos multimedia que permitan llevar a cabo diversas actividades pedagógicas de forma adecuada. Para dar inicio al subproceso, se realiza una petición al encargado de la instalación de equipos multimedia. Cuando el tiempo de uso pactado finaliza el empleado verifica la integridad de los equipos y los desinstala. En cuanto al registro de la información, no se realizan documentos donde se deje constancia sobre la solicitud y devolución del insumo.

\subsubsection{Diagnóstico estratégico}

Teniendo en cuenta la descripción de los procesos, se realizó un diagnóstico estratégico de los procesos con el fin de determinar las condiciones en las cuales se llevan a cabo:

Identificación de necesidades institucionales en cuanto a recursos y servicios: La realización de una reunión de autoevaluación institucional requiere que el coordinador de a conocer a los docentes un documento donde se detalle la fecha, el sitio, y la razón de la reunión. El suministro de esta información es registrado en papel sin tener en cuenta alguna constancia de recibido por parte del cuerpo docente, lo cual influye de forma negativa la verificar si la información fue distribuida adecuadamente.

De la misma forma, en la reunión se utiliza el papel como medio para el registro del Plan de Mejoramiento Institucional, lo cual es una dificultad debido a que se invierte tiempo de la reunión en la elaboración de guías, se generan demoras en detección de necesidades a nivel institucional, y algunas de estas podrían no ser integradas al plan de mejoramiento institucional.

Elaboración del proyecto de presupuesto anual institucional: Este procedimiento se registra en papel y se almacena en archivadores, lo cual atenta contra la seguridad de la información debido a las pérdidas que pueden generarse por el desgaste del papel. Lo anterior, dificulta la elaboración oportuna del proyecto, e interfiere con la secuencia organizativa lo cual incide de forma negativa en el control, seguimiento, evaluación del proyecto. Además, afectar al cuerpo estudiantil porque se puede realizar una mala administración de los recursos de la institución.

Compra de bienes y servicios: Esta actividad se realiza informalmente y como constancia se guardan, de forma desorganizada, facturas y soportes de compra en archivadores localizados en la oficina de rectoría. Esto puede generar irregularidades en cuanto a la inversión en bienes y servicios, lo cual afecta de forma negativa al control 
de bienes de la entidad. Lo descrito, es una razón por la cual los documentos deben ser digitalizados y organizados con el fin de evitar inconvenientes en el futuro. Además, existen diversas ocasiones en las cuales el insumo solicitado es recibido por algún trabajador del área administrativa, la secretaria o el vigilante, quienes pueden omitir el proceso de verificación del producto por lo cual se genera el riesgo de recibir algo diferente a los solicitado.

Recibo y control de bienes en la Institución: La información se almacena en cuaderno los cuales son guardados en archivadores localizados en la oficina de rectoría. Esto supone demoras en actividades desempeñadas por el contador, debido a que el empleado debe utilizar parte de su tiempo laboral al desplazarse de su sitio de trabajo a la oficina de rectoría con el fin de obtener información relacionada con el subproceso. El transporte de documentos de un sitio a otro incrementa el desgaste físico la posibilidad de extravío, por lo cual esto se convierte en una amenaza para la administración de recursos de la entidad. De esta forma, es necesario establecer planes de digitalización periódica con el fin de realizar respaldos en caso que se materialice algún inconveniente y facilitar el acceso a la información por parte del contador.

Entrega de insumos a docentes y directivos: En diversas ocasiones no se realizan registros de los préstamos de insumos realizados a los docentes. Lo anterior, se refleja, principalmente, en situaciones donde se solicita el préstamo de insumos que tiene un valor financiero bajo, lo cual influye de forma negativa en la administración de los recursos de la institución. De esta forma, es necesario implementar estrategias enfocadas en el control de insumos con el fin de prevenir inconvenientes que dificulten el desarrollo de actividades que requieran de la disponibilidad del insumo.

Gestión de solicitud de recursos tecnológicos para actividades pedagógicas: Este subproceso de realiza de manera informal sin dejar constancia de la hora, la cantidad y la razón del préstamo, lo cual puede ser una dificultad para encontrar una solución en caso de pérdida o daño del dispositivo prestado.

\subsubsection{Diagnóstico técnico}

Mediante este diagnóstico se describe la infraestructura de red y tecnología con el fin de identificar aspectos técnicos que necesiten mejorar.

\section{Análisis de infraestructura de la red del área administrativa}

Se determinó que la institución cuenta un canal de internet dedicado con una velocidad de 50Mbs, el cual se distribuye a través del router principal ubicado en la entrada de la institución. De este dispositivo se derivan dos conexiones que suministran el servicio a la oficina de rectoría y la oficina del contador. La primera, se llega directo a un switch Tp-link Tl-sf1005d el cual distribuye el internet hacia 3 equipo de cómputo de la oficina, donde se establece una conexión vía Ethernet a la computadora del rector y dos conexiones WIFI para los equipos de la secretaria y el coordinador. En cuanto la conexión encargada de proporcionar internet a la oficina del contador, se utiliza un router de las mismas características, el cual se encarga de trasmitir internet mediante un cable de red al equipo informático utilizado por el contador. No se encontraron dificultades técnicas que puedan generar inconvenientes en la operación de los procesos del área administrativa.

\section{Análisis de la infraestructura de equipos de cómputo del área administrativa}

En el caso de la oficina de rectoría se identificaron dos computadoras portátiles pertenecientes al coordinador y la secretaria, y un equipo de escritorio utilizado por el rector. Por otra parte, en la oficina del contador se encontró un equipo de cómputo de escritorio en el cual trabaja el empleado. No se encontraron dificultades técnicas que puedan generar inconvenientes en la operación de los equipos informáticos. 


\subsubsection{Análisis diagnóstico}

Se realizó una Matriz DOFA, a través de la cual se identificaron diferentes aspectos como las fortalezas y debilidades de la institución, como se aprecia en la Tabla 2.

\section{Tabla 2}

Matriz DOFA

\begin{tabular}{|c|c|}
\hline Categoría & Descripción \\
\hline \multirow{9}{*}{ Debilidades } & $\begin{array}{l}\text { 1.Poco uso de herramientas informáticas que apoyen las actividades relacionadas con la elaboración } \\
\text { del proyecto de presupuesto anual }\end{array}$ \\
\hline & $\begin{array}{l}\text { 2.No se utiliza la informática para la proyección y seguimiento del proyecto de presupuesto anual del } \\
\text { fondo de servicio educativo }\end{array}$ \\
\hline & $\begin{array}{l}\text { 3.Poca gestión dirigida a la renovación de recursos tecnológicos que apoyan las actividades } \\
\text { administrativas. }\end{array}$ \\
\hline & 4.Poca organización en la información almacenada en los equipos de cómputo del área administrativa \\
\hline & $\begin{array}{l}\text { 5.Desaprovechamiento de herramientas como el internet para la difusión de información hacia la } \\
\text { comunidad académica }\end{array}$ \\
\hline & 6.Manejo poco eficiente de bienes requeridos en el área administrativa de la institución \\
\hline & $\begin{array}{l}\text { 7.Cultura organizacional débil con relación a la utilización de la informática } \\
\text { como herramienta de apoyo a las funciones administrativas }\end{array}$ \\
\hline & $\begin{array}{l}\text { 8.El almacenamiento de la información administrativa se realiza de manera rudimentaria, utilizando } \\
\text { papel y archivadores físicos }\end{array}$ \\
\hline & $\begin{array}{l}\text { 9.No existe protocolo de seguimiento para el proceso de compra, adquisición y recibo de bienes y } \\
\text { servicios }\end{array}$ \\
\hline \multirow{4}{*}{ Oportunidades } & $\begin{array}{l}\text { 1.Recursos disponibles en el mercado sobre nuevas tendencias basadas en aplicaciones de dominio } \\
\text { público }\end{array}$ \\
\hline & $\begin{array}{l}\text { 2.Jornadas pedagógicas para los usuarios del área administrativa sobre las ventajas de incluir las TIC en } \\
\text { los procesos administrativos }\end{array}$ \\
\hline & 3.Expertos en informática y aplicativos para mejorar procesos institucionales \\
\hline & $\begin{array}{l}\text { 4.Mejoramiento y transformación de los procesos administrativos por acogimiento de las tecnologías } \\
\text { informáticas }\end{array}$ \\
\hline \multirow{4}{*}{ Fortalezas } & 1.Existe en la institución una infraestructura a nivel de red Internet \\
\hline & 2.Inversión en equipos de cómputo y redes que apoyan el crecimiento de la Institución \\
\hline & $\begin{array}{l}\text { 3.La institución cuenta con el servicio de un profesional en contabilidad facilitando la gestión de } \\
\text { recursos }\end{array}$ \\
\hline & $\begin{array}{l}\text { 4.Existe conciencia sobre la optimización de los recursos financieros por parte de los entes } \\
\text { administrativos de la intuición }\end{array}$ \\
\hline \multirow{2}{*}{ Amenazas } & $\begin{array}{l}\text { 1.Falta de presupuesto del fondo de servicio educativo para el financiamiento de proyectos específicos } \\
\text { de infraestructura tecnológica }\end{array}$ \\
\hline & $\begin{array}{l}\text { 2.Rápida aceleración de la tecnología ocasionado desactualización en las herramientas informáticas } \\
\text { existentes en la institución }\end{array}$ \\
\hline
\end{tabular}

Fuente: Autores

Lo anterior indica que existen dificultades en diferentes procesos de la institución, como la falta de uso de herramientas informáticas que faciliten el control del proyecto de presupuesto anual, y la carencia en cuanto al uso de internet para el suministro de información, por lo cual deben implementarse estrategias que permitan reducirlas y contribuir al incremento de la efectividad de las actividades en cuanto al manejo de la información desde un punto de vista administrativo. Lo anterior, guarda similitud con (Pimenta \& Minoro, 2019), donde se indica que el manejo adecuado de la información es un aspecto significativo para el funcionamiento de instituciones educativas, lo cual resalta la importancia de la aplicación de estrategias facilitan la gestión de la información en diversas entidades laborales como las instituciones educativas. 
Asimismo, existen características que pueden aprovecharse en la institución para lograr mejoras en las actividades de naturaleza administrativa, como la existencia de una infraestructura de red y la inversión en la compra de equipos de informáticos que permiten el desarrollo de la institución a nivel competitivo. Teniendo en cuenta lo descrito, se realizó una Matriz de Impacto (ver tabla 3) con el fin de evaluar la prioridad de atención requerido por los ítems obtenidos mediante la Matriz DOFA.

Tabla 3

Matriz de impacto

\begin{tabular}{|c|c|c|c|}
\hline \multirow{2}{*}{ Debilidades } & \multicolumn{3}{|c|}{ Impacto } \\
\hline & Alto & Medio & Bajo \\
\hline 1 & $x$ & & \\
\hline 2 & $x$ & & \\
\hline 3 & & $x$ & \\
\hline 4 & & $x$ & \\
\hline 5 & $x$ & & \\
\hline 6 & $X$ & & \\
\hline 7 & $x$ & & \\
\hline 8 & & $x$ & \\
\hline 9 & $x$ & & \\
\hline \multirow{2}{*}{ Oportunidades } & \multicolumn{3}{|c|}{ Impacto } \\
\hline & Alto & Medio & Bajo \\
\hline 1 & $x$ & & \\
\hline 2 & & $x$ & \\
\hline 3 & $x$ & & \\
\hline 4 & $\mathrm{X}$ & & \\
\hline \multirow{2}{*}{ Fortalezas } & \multicolumn{3}{|c|}{ Impacto } \\
\hline & Alto & Medio & Bajo \\
\hline 1 & $x$ & & \\
\hline 2 & & $x$ & \\
\hline 3 & & $x$ & \\
\hline 4 & & $x$ & \\
\hline \multirow{2}{*}{ Amenazas } & \multicolumn{3}{|c|}{ Impacto } \\
\hline & Alto & Medio & Bajo \\
\hline 1 & & $x$ & \\
\hline 2 & & $x$ & \\
\hline
\end{tabular}

Fuente: Autores

De esta forma se determinó que existen elementos a los cuales debe prestarse atención con alta prioridad, lo cual sirvió como base la establecer estrategias que permitan reducir dificultades en el área administrativa de la institución, como se muestra en la Tabla 4.

Tabla 4

Estrategias propuestas

\begin{tabular}{|c|c|}
\hline$\#$ & Estrategia \\
\hline 1 & $\begin{array}{c}\text { Implementar jornadas de capacitación sobre el uso de las TIC y } \\
\text { principios técnicos, dirigidas a funcionarios de la división } \\
\text { administrativa de la Institución, y concientizarlos al uso } \\
\text { eficiente de las mismas. }\end{array}$ \\
\hline 2 & $\begin{array}{c}\text { Gestión y mantenimiento de los equipos informáticos } \\
\text { empleados para llevar a cabo los procesos en el área } \\
\text { administrativa de la Institución. }\end{array}$ \\
\hline
\end{tabular}




\begin{tabular}{|c|c|}
\hline 3 & $\begin{array}{c}\text { Realizar la digitalización de toda la información manejada en el } \\
\text { área administrativa que se encuentra en papel. }\end{array}$ \\
\hline 4 & $\begin{array}{c}\text { Implementar sistemas de información como solución a las } \\
\text { necesidades encontradas en el área administrativa de la } \\
\text { Institución. }\end{array}$ \\
\hline 5 & $\begin{array}{c}\text { Implementar jornadas de capacitación sobre el funcionamiento } \\
\text { de los sistemas informáticos que se implementarán para el } \\
\text { manejo de los procesos administrativos }\end{array}$ \\
\hline 6 & $\begin{array}{c}\text { Gestionar y organizar la información que pueda encontrarse de } \\
\text { manera virtual en equipos informáticos del área administrativa }\end{array}$ \\
\hline
\end{tabular}

Fuente: Autores

Las estrategias establecidas fueron sometidas al análisis de los directivos dela institución con el fin de determinar la pertinencia de las mismas.

\subsection{Elaboración del plan estratégico informàtico PEI}

En esta etapa se elaboró el PEI, teniendo en cuenta estrategias planteadas en la etapa anterior, con la finalidad de impulsar las capacidades de TIC requeridas para lograr mejoras en los procesos del área administrativa de la institución e incrementar el nivel competitivo. Para la creación del PEI se establecieron proyectos que abordan diferentes dificultades encontradas, entre los más relevantes se destacan Capacitación técnica dirigida a funcionarios administrativos en TIC, Organización documental y digitalización de la información, e Implementación de un aplicativo de inventario para la gestión de recursos, bienes y servicios institucionales.

Respecto al proyecto Capacitación técnica en TIC dirigida a funcionarios administrativos, consiste en la aplicación de un plan de capacitación técnica basado en las TIC dirigido a los empleados del área administrativa de la institución, donde se establecieron estrategias que sirven de base para el PEI y acciones que funcionan como apoyo para la implementación de las estrategias planteadas, como se observa en la Tabla 5.

Tabla 5

Estrategias y acciones para la capacitación técnica en en TIC dirigida a funcionarios administrativos

\begin{tabular}{|c|}
\hline \begin{tabular}{c} 
Estrategias \\
\hline $\begin{array}{c}\text { Formar al personal del área administrativa en el manejo de las herramientas } \\
\text { informáticas que serán implementadas }\end{array}$ \\
\hline $\begin{array}{c}\text { Capacitación continua al personal administrativo para fortalecer la cultura } \\
\text { institucional para futuras implementación de aplicativos informáticos }\end{array}$ \\
\hline Acciones \\
\hline Selección y preparación de los participantes y de los expertos a cargo de la \\
capacitación \\
\hline Preparación y organización de la logística de la capacitación \\
\hline Preparación y aplicación de las actividades de seguimiento en el plan de \\
capacitación
\end{tabular} \\
\hline
\end{tabular}

Fuente: Autores

Lo anterior, permite crear conciencia sobre la relevancia de las TIC y se convierte en un factor que estimula el uso de este tipo de tecnologías para el logro de mejoras a nivel institucional. Con la implementación de capacitaciones técnicas los trabajadores pueden mejorar sus habilidades respecto al manejo de nuevas tecnologías, lo cual contribuye al desempeño de las actividades administrativas como mayor grado de efectividad. Esto se complementa con (Jung \& Shin, 2015) donde se planea que cuando los empleados 
administrativos de una institución educativa no reciben suficientes oportunidades que contribuyan al crecimiento profesional, el incremento de la productividad podría convertirse en una tarea difícil. Por ello, resulta relevante la aplicación de jornadas educativas, como charlas y capacitaciones, donde el funcionario puede adquirir conocimientos útiles para el desempeño de sus labores.

Por otra parte, el proyecto Organización documental y digitalización de la información consiste en la aplicación de un plan a través del cual se realice una organización documental y la información manipulada en la parte administrativa de la institución sea almacenada de forma digital. Para la creación de este proyecto se implementaron diferentes estrategias y acciones, como se muestra en la Tabla 6.

\section{Tabla 6}

Estrategias y acciones para la organización documental y digitalización de la información

\begin{tabular}{|c|}
\hline $\begin{array}{c}\text { Estrategias } \\
\text { Contratar a personal con conocimiento para digitalizar la } \\
\text { información registrada en papel }\end{array}$ \\
\hline $\begin{array}{c}\text { Almacenar la información en un servicio de Nube de uso } \\
\text { exclusivo del personal administrativo }\end{array}$ \\
\hline $\begin{array}{c}\text { Organizar la información de manera seccionada para facilitar el } \\
\text { acceso en caso de necesitarla }\end{array}$ \\
\hline Acciones \\
\hline $\begin{array}{c}\text { Organizar la información utilizando medios ofimáticos para } \\
\text { almacenarla de modo virtual en un servicio de nube }\end{array}$ \\
\hline
\end{tabular}

Fuente: Autores

De esta forma, se tiene un control adecuado sobre la gestión de la información, se reducen riesgos asociados al deterioro del papel, y se incrementa la eficiencia en diferentes procesos debido a que la información cuenta con mayor disponibilidad.

Por último, el proyecto Implementación de un aplicativo de inventario para la gestión de recursos, bienes y servicios institucionales fue definido con el fin de mejorar el control y seguimiento de en cuanto al manejo de los recursos de la institución disponibles en bodega. Este proyecto el establecimiento de las estrategias y acciones planteadas en la Tabla 7.

\section{Tabla 7}

Estrategias y acciones para la implementación de un aplicativo de inventario para la gestión de recursos, bienes y servicios institucionales

\begin{tabular}{|c|}
\hline Estrategias \\
\hline $\begin{array}{c}\text { Implementar un aplicativo de inventario para la gestión de } \\
\text { recursos, bienes y servicios }\end{array}$ \\
\hline Acciones \\
\hline $\begin{array}{c}\text { Adquirir un software para la realización de inventario y registro } \\
\text { en la gestión de bienes y servicios }\end{array}$ \\
\hline $\begin{array}{c}\text { Almacenar los datos como documento editable en línea, para así } \\
\text { poder acceder a él de manera rápida y a la vez poder manipular } \\
\text { su información oportunamente }\end{array}$ \\
\hline
\end{tabular}

Fuente: Autores

Lo anterior, reducir la informalidad en las actividades de recibo, entrega y registro de bienes en bodega, debido a que el empleado a cargo de la bodega puede registrar información de manera fácil y rápida sobre el solicitante, 
el tiempo y el motivo del préstamo. De esta forma se puede reducir el extravío de insumos prestados a docentes y evitar la inversión innecesaria de recursos financieros en la compra de nuevos bienes.

\subsection{Programación de actividades}

En esta etapa no se obtuvieron resultados relevantes debido a que la asignación de fechas y recursos para los proyectos determinados en la etapa anterior es responsabilidad de los directivos de la institución analizada.

\section{Conclusiones}

De acuerdo a los resultados obtenidos se concluyó lo siguiente:

La digitalización de la información administrativa de la en la institución permite incrementar la eficiencia de los procesos administrativos debido a que se puede acceder a la información de forma fácil, rápida y organizada;

La contratación de un servicio de almacenamiento en la nube permite que los empleados accedan desde el equipo de cómputo ubicado en el lugar de trabajo asignado por la institución, sin necesidad de desplazarse a los archivadores encontrados en la oficina de rectoría, lo cual supone una reducción en la demora al ejecutar actividades del área administrativa;

Se disminuyen riesgos de pérdida o de información administrativa debido a que se elimina el papel como principal fuente de almacenamiento, el cual es propenso al deterioro; y

Con la implementación de una herramienta software que permita el inventario de los recursos, bienes y servicios de la institución se puede tener un buen control la administración del proyecto de prepuesto anual debido a que el aplicativo sirve como base para el uso oportuno del presupuesto.

\section{Referencias bibliográficas}

Alamri, S., Almutiri, N., Ballahar, H., \& Zafar, A. (2016). Strategic Information System Planning: A Case Study of a Service Delivery Company. International Advanced Research Journal in Science, Engineering and Technology, 3(5), 78-84.

Almasri, A., Talla, S., Abu-Naser, S., \& Shobaki, M. (2018). The Organizational Structure and its Role in Applying the Information Technology Used In the Palestinian Universities- Comparative Study between Al-Azhar and the Islamic Universities. International Journal of Academic and Applied Research, 2(6), 1-22.

Andrew, R. (1996). Estrategias y sistemas de información. Barcelona : McGraw-Hill.

Cook, J. (2017). Social class bias: a phenomenological study. The journal of couselor preparation and supervision, 9(1).

Hernández, R., Fernández, C., \& Baptista, P. (2014). Metodología de la investigación. México: Mc Graw Hill Education.

Jung, J., \& Shin, J. (2015). Administrative staff members' job competency and their job satisfaction in a Korean research university. Studies in Higher Education, 40(5), 881-901. doi:10.1080/03075079.2013.865161

Kitsios, F., Kamarituo, M., Madas, M., Fouskas, K., \& Manthou, V. (2020). Information systems strategy in SMEs:critical factors of strategic planningin logistics. Kybernetes , 49(4), 1197-1212. 
Kumar, S., \& Shardhi, T. (2019). Performance Measure of Classifier for Prediction of Healthcare Clinical Information. First International Conference on Sustainable Technologies for Computational Intelligence. 1045, págs. 289-301. Singapore: Springer. doi:10.1007/978-981-15-0029-9_23

Labas, I., Darabos, E., \& Nagy, O. (2016). COMPETITIVENESS - HIGHER EDUCATION. Studia Universitatis Economics Series, 26(1), 11-25. doi:10.1515/sues-2016-0002

Martelo, R., Ponce, A., \& Acuña, F. (2015). Guía Metodológica para el Diseño de un Plan. Guía Metodológica para , 9(1), 91-98.

Pimenta, A., \& Minoro, J. (2019). Determination of the turning point of cache efficiency in computer networks with logic E. Orocedia Computer Science , 159, 1182-1189. doi:10.1016/j.procs.2019.09.287

Sarif, S., Rahman, N., Yunus, S., \& Rahamn, N. (2018). Strategic Information System Planning (SISP) Success: A Case Study. International Conference on Kansei Engineering and Emotion Research. Singapore: Springer. doi:10.1007/978-981-10-8612-0_72

Shahid, M., \& Luo, B. (2019). Prediction of educational institution using predictive analytic techniques . Education and Information Technologies, 24, 1469-1483. doi:doi.org/10.1007/s10639-018-9827-y

Esta obra está bajo una Licencia Creative Commons Attribución-NoCommercial 4.0 International

\section{(cc) EY-NC}

